\title{
The Role of Cholecystokinin 1 Receptor in Prolactin Inhibited Gastric Emptying of Male Rat
}

\author{
Full-Young Chang, ${ }^{1 *}$ Ching-Liang Lu, ${ }^{1}$ Tseng-Shing Chen ${ }^{1}$ and Paulus S Wang ${ }^{2}$ \\ ${ }^{1}$ Environmental Heath and Safety Office and Division of Gastroenterology, Taipei Veterans General Hospital, Taipei, Taiwan; and ${ }^{2}$ Department \\ of Physiology, National Yang-Ming University School of Medicine, Taipei, Taiwan
}

\section{Background/Aims}

Prolactin (PRL) is essential for the lactating mammals, while cholecystokinin (CCK) does inhibit gastric emptying (GE). Present study attempted to determine whether both peptides interacted on the male rat GE, particularly the role of putative CCK1 receptor.

\section{Methods}

Acute hyperprolactinemia of male rats was induced by the intraperitoneal injection of ovine PRL (oPRL) in several divided doses 15 minutes before motility study. Rat chronic hyperprolactinemia was induced by the graft of 2 pituitary glands into the capsule of left kidney, while control rats received cerebral cortex graft only. Motility study was conducted 6 weeks later after graft surgery. Fifteen minutes after the intragastric feeding of radiochromium, rat was sacrificed to measure GE via the distribution of radioactivities within stomach and intestine. Among the CCK1 receptor blocking study using lorglumide, rats were divided to receive the regimens in terms of oPRL-vehicle plus lorglumide-vehicle, oPRL plus lorglumide-vehicle, oPRL-vehicle plus lorglumide and OPRL plus lorglumide. Plasma CCK level was measured using a homemade radioimmunoassay kit.

\section{Results}

Compared to vehicle treatment, acute hyperprolactinemic rats under highest dose $(2.0 \mathrm{mg} / \mathrm{kg})$ of oPRL treatment showed delayed GE $(70.6 \% \pm 3.0 \%$ vs $42.1 \% \pm 6.6 \%, P<0.05)$. Chronic hyperprolactinemic rats under graft surgery also showed inhibited GE $(70.5 \% \pm 1.7 \%$ vs $54.5 \% \pm 4.7 \%, P<0.05)$. Both models finally obtained elevated plasma CCK levels $(P<$ 0.05). Lorglumide itself did not influence $G E$, however, delayed GE under oPRL treatment was restored following the concomitant lorglumide treatment.

\section{Conclusions}

Our study suggests that PRL may delay male rat GE via a mechanism of endogenous CCK activation involving the peripheral CCK1 receptor.

\section{(J Neurogastroenterol Motil 2012;18:385-390)}

\section{Key Words}

Cholecystokinin; Gastric emptying; Lorglumide; Prolactin

Received: May 4, 2012 Revised: June 14, 2012 Accepted: June 19, 2012

(c) This is an Open Access article distributed under the terms of the Creative Commons Attribution Non-Commercial License (http://creativecommons org/licenses/by-nc/3.0) which permits unrestricted non-commercial use, distribution, and reproduction in any medium, provided the original work is properly cited.

*Correspondence: Full-Young Chang, MD

Environmental Heath and Safety Office, Taipei Veterans General Hospital, 201 Shih-Pai Road, Section 2, Taipei 11217, Taiwan Tel: +886-2-28757308, Fax: +886-2-28757310, E-mail: changfy@vghtpe.gov.tw

Financial support: This study was sponsored from a grant of National Science Council, Republic of China (NSC 89-2314-B-075-070).

Conflicts of interest: None. 


\section{Introduction}

The physiological mechanisms in regulation of gastric emptying (GE) are complex. Basically, stomach delivery or GE involves antral contraction, pyloric relaxation and finally duodenal accommodation to accept the coming luminal contents. ${ }^{1}$ Apart from myogenic ability to provide motor power, both neural and humoral mechanisms in the high levels are essential in modulating GE. ${ }^{2}$ Even sexual hormones have been the mediators in controlling gastric motility. For example, estrogen inhibits GE of ovariectomized rats, whereas progesterone enhances their GE. ${ }^{3}$ Similar to oxytocin, prolactin (PRL) is one of the pituitary gland secretagogues which is mainly inhibited by the hypothalamic dopamine. ${ }^{4}$ Apart from breast tissue, PRL receptors are also identified in the gastrointestinal (GI) tract. Perhaps PRL mainly coordinates the metabolism, growth and cellular differentiation of GI tract. ${ }^{5}$ Likewise, we have observed the higher the blood PRL level in the lactated female rats the rapid the GE of these animals, while the small intestinal lengths of lactating rats correlated well with their blood PRL levels. ${ }^{6}$ Cholecystokinin (CCK) is a famous gut peptide to display the physiological functions in terms of stimulation of pancreatic enzyme secretion, gall bladder contraction, inhibited GE and bowel motility leading to the suppressed food intake and even the regulation of other gut hormones, eg insulin, glucagon and pancreatic polypeptide. ${ }^{7-9}$ It is also involved in the hypothalamic-pituitary-adrenal axis since CCK active form releases PRL and vasopressin in animals and humans. ${ }^{10}$ It probably means the closed interaction of CCK and PRL. We have demonstrated that oxytocin inhibited male/female rat GE is likely activated via a mechanism involving CCK stimulation on one of its receptors, the CCK $1 .{ }^{11}$ Besides, an in vitro study also pointed out that oxytocin inhibited duodenal longitudinal muscle contraction via the CCK release from myenteric plexus neurons. ${ }^{12} \mathrm{We}$ are interested to know whether CCK may also be involved in the PRL mediated GI motility. Using the acute and chronic hyperprolactinemic animal models, the purpose of present study was attempted to elucidate whether the regulation of PRL on rat GE is also mediated via CCK pathway.

\section{Materials and Methods}

\section{Animals and Gastric Emptying Study}

Adult Sprague-Dawley male rats, 3-4 months old, weighing
300-400 g, were obtained from the Animal Center of National Yang-Ming University. The protocol was approved by the Institutional Animal Care and Use Committee of National Yang-Ming University. All the animals were cared in compliance with the Principles of Laboratory Animal Care and the Guide for the Care and Use of Laboratory Animals published by the National Science Council, Republic of China. They were housed under the controlled conditions of light (06:00-20:00), humidity and temperature $\left(22 \pm 1^{\circ} \mathrm{C}\right)$. Standard laboratory chow and water were available ad libitum. Liquid GE was the representation of present GI motility study. It measured the propulsion of a non-absorbable marker within the gut after an orogastric instillation. Briefly, the feeding procedure was achieved with a temporarily placed orogastric catheter (ID: $1.67 \mathrm{~mm}, \mathrm{OD}$ : 2.42 mm, PE-205; Clay-Adams, Parsippany, NJ, USA). The motility marker of this nutrient-free liquid was $\mathrm{Na}^{51} \mathrm{CrO}_{4}$ (Dupont, NEN Research Products, Boston, MA, USA) with a radioactivity of $0.5 \mathrm{mCi} / \mathrm{mL}$. All the studied rats were kept in the conscious state and the feeding amount of radiochromium was adjusted to $3 \mathrm{~mL} / \mathrm{kg}$. Then the animals were instantly sacrificed with a guillotine 15 minutes later after the successful feeding. Blood was collected to measure plasma CCK levels while the entire stomach and small intestine were carefully removed. The radioactivities of divided stomach and small intestinal segments were measured using a gammacounter (1277 GammaMaster, Pharmacia, Turku, Finland). GE is the percentage of small intestinal radioactivity divided by the total radioactivity recovered from entire stomach plus small intestine. ${ }^{13}$

\section{Induction of Hyperprolactinemia}

Acute hyperprolactinemia was induced by the intraperitoneal (i.p.) injection of ovine PRL (oPRL, Sigma, St. Louis, MO, USA $)$ in the doses of $0.5(n=6), 1(n=6)$ and $2 \mathrm{mg} / \mathrm{kg}(\mathrm{n}=$ $6)$, respectively, 15 minutes before the motility study. This peptide was diluted using the vehicle of deionized water, while control rats $(n=6)$ only received vehicle injection. On the other hand, the induction of chronic hyperprolactinemia was followed in accordance with the original of Everett and ours. ${ }^{14,15}$ Briefly, an incision was made in the rat left flank to expose left kidney under the light ether anesthesia. A slit in the renal capsule was made in the graft rat to receive an implantation of 2 anterior pituitary glands ( $\mathrm{AP}, \mathrm{n}=10$ ) in the space beneath. Control rats only received the similar amount of brain cortex $(\mathrm{CX}, \mathrm{n}=10)$ under the similar surgery. Liquid GE study was conducted 6 weeks later following either AP or CX graft surgery accordingly. 


\section{Lorglumide Blocking Study}

Another new group of acute hyperprolactinemic rat model underwent CCK blocking study using the combined i.p. administration of oPRL and lorglumide, a very specific CCK1 receptor antagonist. $^{16}$ Lorglumide sodium (Research Biochemicals International Co., Natick, MA, USA) was diluted using the vehicle of deionized water. The combined treatment was administered 15 minutes before GE measurement using lorglumide dose in accordance with our previous studies. ${ }^{11,16}$ Lorglumide blocked rats were divided into 4 groups to receive the regimens: oPRL-vehicle plus lorglumide-vehicle $(\mathrm{n}=7)$, oPRL-vehicle plus lorglumide $10 \mathrm{mg} / \mathrm{kg}$ ( $\mathrm{n}=7$ ), oPRL $1 \mathrm{mg} / \mathrm{kg}$ plus lorglumide-vehicle $(\mathrm{n}=7)$ and $\mathrm{oPRL} 1 \mathrm{mg} / \mathrm{kg}$ plus lorglumide 10 $\mathrm{mg} / \mathrm{kg}(\mathrm{n}=7)$, respectively.

\section{Plasma Processing}

After the decapitation, rat blood samples were collected and mixed with EDTA (1 $\mathrm{mg} / \mathrm{mL}$ of blood) and aprotinin (5 $\mathrm{TIU} / \mathrm{mL}$ of blood). Plasma was immediately obtained by a centrifugation at $1,000 \mathrm{~g}$ for 30 minutes at $4{ }^{\circ} \mathrm{C}$, then the samples were acidified with an equal volume of $1 \%$ trifluoroacetic acid (Buffer A) and then centrifuged at 2,600 g for 20 minutes at $4^{\circ} \mathrm{C}$. A SEP-PAK C18 cartridge (Waters Associates, Milford, MA, USA) was equilibrated by $60 \%$ acetonitrile in $1 \%$ TFA $(1 \mathrm{~mL}$, Buffer B) and followed by Buffer A (3 mL, 3 times). The plasma solution was loaded onto the pretreated $\mathrm{C} 18$ cartridge. After ap-

A

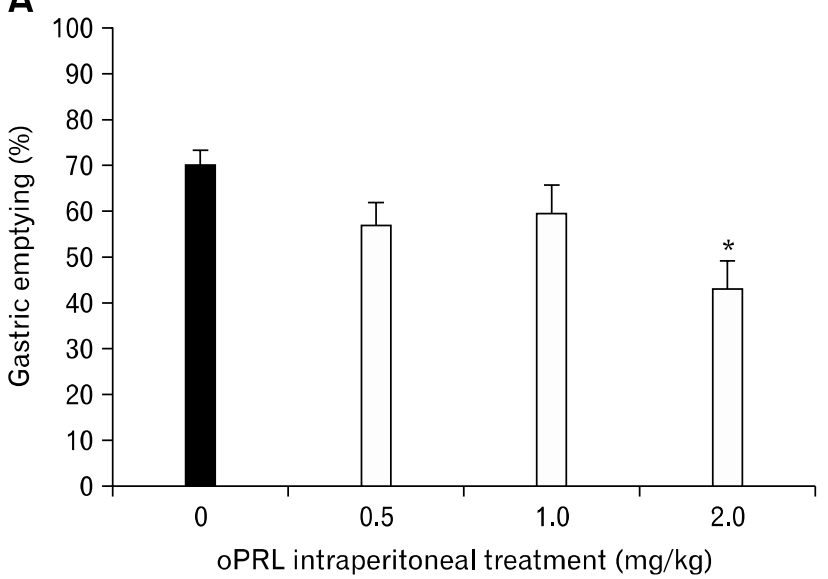

plication of plasma the cartridge was slowly washed with $3 \mathrm{~mL}$ Buffer A twice and the peptide slowly eluted with $3 \mathrm{~mL}$ Buffer B. The eluant was collected and evaporated in a speed vacuum concentrator (Salvant Instruments, Farmingdale, NY, USA). The dried samples were maintained at $-80^{\circ} \mathrm{C}$ and subsequently reconstituted with an assay buffer before radioimmunoassay (RIA).

\section{Plasma Cholecystokinin (CCK) Analysis}

The CCK level in the extracted sample was measured using the similar homemade RIA we had measured previously. ${ }^{11}$ Briefly, a known amount of unlabeled CCK adjusted to a total volume of $0.3 \mathrm{~mL}$ by $0.1 \%$ gelatin-phosphate buffer saline was incubated with $0.1 \mathrm{~mL}$ of CCK antiserum $(1: 2,000)$ which was diluted with normal rabbit serum and $100 \mathrm{~mL}$ of $\left[{ }^{3} \mathrm{H}\right] \mathrm{CCK}$ $\left(\sim 8,000 \mathrm{cpm}\right.$; Amersham, Bucks, UK) at $4^{\circ} \mathrm{C}$ for 24 hours. Triplicate standard curves with 6 points ranging from 1 to 1,000 pg were included in each assay, while unknown samples were assayed in one sample. A total of $200 \mathrm{~mL}$ anti-rabbit-gamma-globulin was then added with further incubation at $4^{\circ} \mathrm{C}$ for 24 hours. At the end of incubation the assay tubes were centrifuged at 1,000 $\mathrm{g}$ for 20 minutes. The pellet was mixed with $400 \mathrm{~mL} 1 \mathrm{~N} \mathrm{NaOH}$ and $80 \mathrm{~mL} 5 \mathrm{~N} \mathrm{HCl}$. The mixture was then mixed with $3 \mathrm{~mL}$ of liquid scintillation fluid (Wallac 1409; Pharmacia, Turku, Finland) before the radioactivity was counted in an automatic counter. The measured sensitivity was 8 pg per assay tube. The intra- and inter-assay coefficients of variation were $3 \%$ and $5 \%$, respectively.

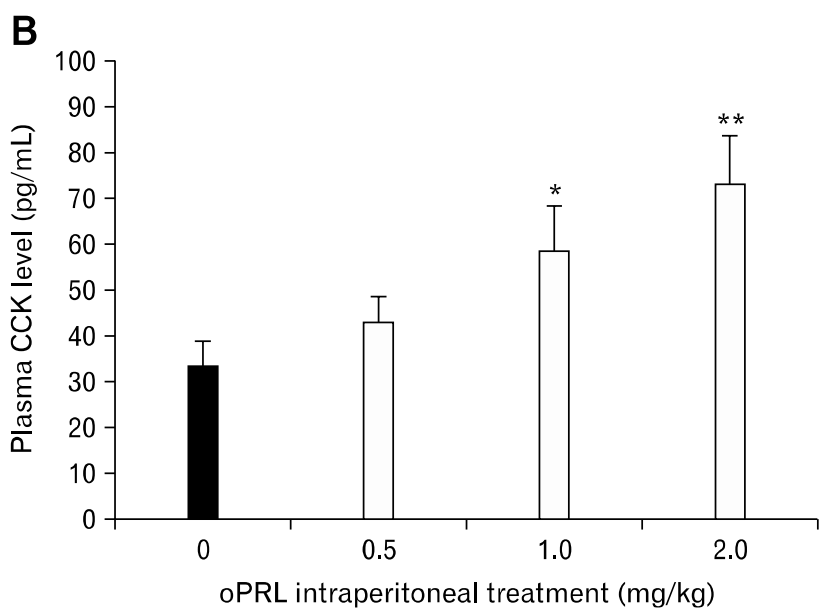

Figure 1. Gastric emptying value and plasma cholecystokinin (CCK) level of acute hyperprolactinemic male rats. (A) Radiochromium feeding measured gastric emptying of rats receiving intraperitoneal treatment of ovine prolactin (oPRL), while control rats only received vehicle treatment ( $\mathbf{\square}$ ). Highest dose of oPRL treatment inhibited gastric emptying $\left({ }^{*} P<0.05\right)$. (B) The radioimmunoassay obtained plasma CCK levels of these rats after oPRL treatment. Higher doses of oPRL elevated plasma CCK levels in a dose dependent manner $\left({ }^{*} P<0.05\right.$ and $\left.* * P<0.01\right)$. Bars above columns are SE. 
A

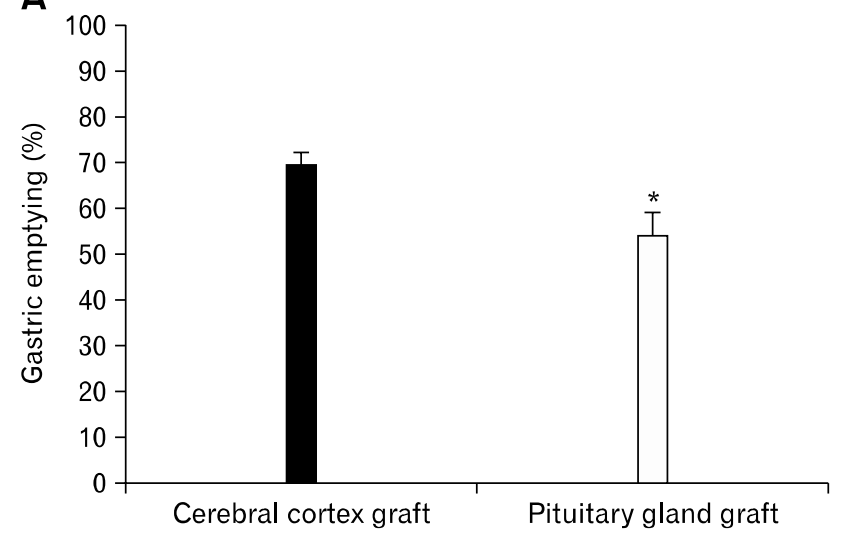

B

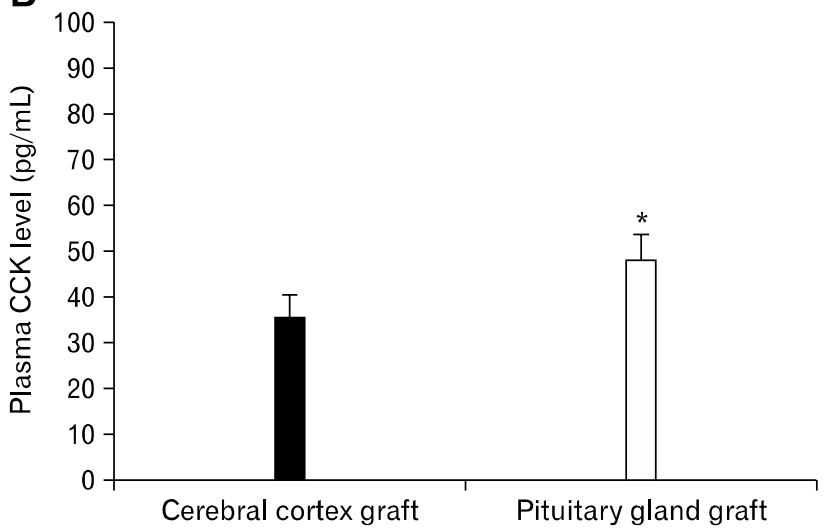

Figure 2. Gastric emptying value and plasma cholecystokinin (CCK) level of chronic hyperprolactinemic male rats. (A) Radiochromium feeding measured gastric emptying of rats receiving brain graft surgery. Chronic hyperprolactinemic rats received pituitary gland graft ( $\square$ ), while control rats only received brain cortex tissue graft $(\square)$. Pituitary gland graft inhibited gastric emptying $(* P<0.05)$. (B) The radioimmunoassay obtained plasma CCK levels of these rats after graft surgery. Pituitary gland graft elevated plasma CCK level $(* P<0.05)$. Bars above columns are SE.

\section{Statistical Methods}

All values were expressed as mean $\pm \mathrm{SE}$. Numerical data were analyzed using a one-way analysis of variance (ANOVA) with Dunnett's post test. A $P$-value of less than 0.05 was considered significant.

\section{Results}

Among the acute hyperprolactinemic model, the measured $\mathrm{GE}$ of rats receiving vehicle treatment which served as control was $70.6 \% \pm 3.0 \%$, while the GEs of rats receiving 0.5 and 1.0 $\mathrm{mg} / \mathrm{kg}$ oPRL treatments were $57.2 \% \pm 5.1 \%$ and $59.3 \% \pm$ $6.3 \%$, respectively (NS). However, the GE $(42.1 \% \pm 6.6 \%)$ of rats with highest dose $(2.0 \mathrm{mg} / \mathrm{kg})$ of oPRL treatment displayed significant inhibition compared to vehicle treatment $(P<0.05$, Fig. 1A). Meanwhile, the RIA measured plasma CCK level of rats receiving vehicle treatment was $33.8 \pm 5.7 \mathrm{pg} / \mathrm{mL}$, while the plasma CCK level of rats receiving low dose $(0.5 \mathrm{mg} / \mathrm{kg})$ of oPRL treatment was $42.4 \pm 5.5 \mathrm{pg} / \mathrm{mL}$ (NS). On the other hand, the rats under higher doses $(1.0$ and $2.0 \mathrm{mg} / \mathrm{kg}$ ) of oPRL treatments showed elevated CCK levels compared to vehicle treated rats as $58.5 \pm 9.4 \mathrm{pg} / \mathrm{mL}$ and $72.4 \pm 11.1 \mathrm{pg} / \mathrm{mL}$, respectively $(P<0.05$ and 0.01 , Fig. $1 B)$. With regard to the chronic hyperprolactinemic model, the measured GE of CXgrafted rats which served as control was $70.5 \% \pm 1.7 \%$, whereas this of AP-grafted rats was significantly inhibited to $54.5 \% \pm$ $4.7 \%(P<0.05$, Fig. 2A). The RIA measured plasma CCK level of CX-grafted control rats was $35.8 \pm 5.1 \mathrm{pg} / \mathrm{mL}$, while

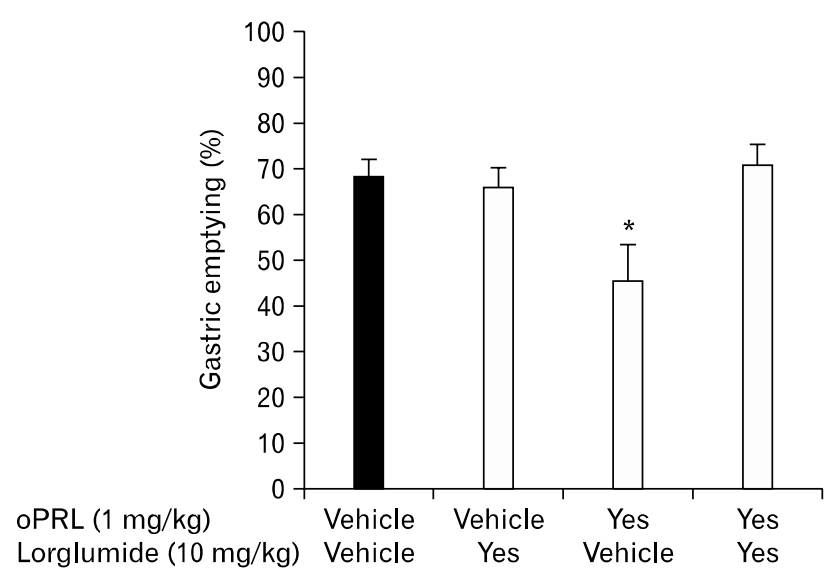

Figure 3. Radiochromium feeding measured gastric emptying of rats receiving intraperitoneal dual treatment of ovine prolactin (oPRL) plus cholecystokinin 1 receptor antagonist-lorglumide. Control rats only received vehicle treatment $(\boldsymbol{\square})$. The oPRL treatment in the lorglumide-vehicle inhibited gastric emptying $(* P<0.05)$. This inhibition was restored when lorglumide was concomitantly treated together. Bars above columns are SE.

AP-graft markedly elevated plasma CCK level to $48.5 \pm 6.3$ $\mathrm{pg} / \mathrm{mL}$ in comparison with control rats $(P<0.05$, Fig. $2 \mathrm{~B})$. Among the CCK blocking study based on dual oPRL and lorglumide treatment, the rats receiving oPRL-vehicle plus lorglumide-vehicle were served as controls showing a GE value of $68.8 \% \pm 3.0 \%$. Lorglumide itself did not have any effect on rat $\mathrm{GE}(66.7 \% \pm 3.9 \%)$. Treatment of oPRL in the absence of lorglumide again markedly inhibited GE to $45.3 \% \pm 7.5 \%(P<$ $0.05)$. However, this kind of inhibited GE was restored up to 
$71.8 \% \pm 4.3 \%$ when lorglumide was concomitantly administrated with oPRL treatment (Fig. 3).

\section{Discussion}

Our study mainly observed that experimentally induced hyperprolactinemic male rats had delayed GE, irrespective of acute oPRL i.p treatment or chronic AP-graft. Although the current study did not simultaneously measure plasma PRL levels in both models, however, our previous studies already confirmed the successful induction of hyperprolactinema with almost 2-fold increase for the male AP-grafted rats. ${ }^{15}$ Among the acute hyperprolactinemic male rats, we did observe their elevated plasma CCK levels following acute oPRL i.p treatment. In addition, we also demonstrated that AP-graft effectively elevated plasma CCK level compared to CX-grafted rats. Accordingly, the elevated plasma CCK level as well as its delayed GE is likely resulted from the hyperprolactinemic induction, irrespectively of acute oPRL i.p. induction or chronic AP-graft. It is unknown whether there are certain PRL receptors closely adherent to the CCK producing cells since the endogenous CCK is produced under such acute and chronic PRL treated models, and current study was not designed to resolve this controversy.

Mammalian lactation is associated with physiological changes in terms of enhanced GE, sped intestinal transit and gut hyperplasia in the lactating rodents, while some of these changes have been the direct effect of PRL. ${ }^{6,17,18}$ Besides, i.p. PRL treatment sped mice GI transit, which suggested the modulation via cholinomimetic pathway. ${ }^{19}$ Perhaps PRL modulates GE via other pathways among the lactated rats. In contrast, our study pointed out that exogenous PRL treatment inhibited male rat GE as well as those of chronic hyperprolactinemic male rats receiving AP graft. We are uncertain whether the gender or lactation difference may account for these discrepant GE results. Unlike lactating female rats showing enhanced GE, we suggest that PRL inhibits male rat GE probably representing the gender specificity. Similarly, Vijayan and McCann ${ }^{20}$ pointed out that proglumide, a nonspecific CCK receptor antagonist, diminished PRL concentration of male rats, whereas this decrease was not observed among the ovariectomized female rats. Gender difference might exist with regard to this physiological response. In addition, literature indicated that exogenous CCK or its agonist treatment induced PRL release in healthy men, ${ }^{10}$ adult male/female sheep, ${ }^{21}$ prepubertal pigs ${ }^{22}$ and female/male rats, ${ }^{11,23}$ and it was likely mediated via vasoactive intestinal polypeptide stimulation partic- ularly in male rats. On the other hand, Karashima et $\mathrm{al}^{24}$ found that intra-ventricularly rather than peripherally injected CCK suppressed PRL release in the male Wistar rats, which was obviously functioning in the hypothalmus. However, Miyake et $\mathrm{al}^{25}$ observed that both $\mathrm{CCK}$ and vasoactive intestinal polypeptide were not responsible for the meal induced PRL release in men. Hence the interaction between CCK administration and PRL release remains debatable. These discrepant physiological results are most likely depended upon the various routes of peptide administration, species difference and methods of measurement. Our study found that both acute and chronic hyperprolactinemic male rats had an elevated plasma CCK level. It probably means that both peptides do not exist a bio-feed mechanism each other since neither exogenous nor endogenous PRL inhibits the CCK release, at least among the male rats.

It is well known that $\mathrm{CCK}$ inhibits GE leading to the satiety sensation in turn to reduce food intake. ${ }^{9,26}$ Two CCK receptor subtypes in terms of CCK1 and CCK2 already exist in the tissues. It is believed that CCK1 receptors are most abundant in the peripheral tissues, whereas $\mathrm{CCK} 2$ receptors are mainly located in the central nervous system. ${ }^{16}$ Accordingly, exogenous CCK administration induced satiety and inhibited GE and CCK antagonists to treat various functional GI disorders are usually mediated via blocking CCK1 receptors. ${ }^{27}$ Our study observed that PRL inhibited GE was associated with elevated plasma CCK level, despite of used models of acute or chronic induction. GE inhibition and plasma CCK elevation were obviously found in the acute oPRL treatment in a dose dependant manner. Lorglumide itself had no role in influencing GE. In addition, its simultaneous administration on the male rats receiving exogenous oPRL treatment restored the inhibited GE. Considering these findings, our results are likely to suggest that PRL delayed GE in male rats was similarly mediated via activated CCK through its peripheral CCK1 receptor pathway as well as the inhibitory ability of oxytocin on rat GE. ${ }^{11}$ Because we did not conduct the blocking of central CCK2 receptor pathway, current study could not resolve whether PRL may additionally inhibit male rat GE via the central CCK2 receptor pathway. In conclusion, present study observed that peripheral oPRL administration or endogenous PRL release using AP graft inhibited male rat GE and elevated plasma CCK level, while this delayed GE was restored using a specific CCK1 receptor antagonist. Perhaps PRL inhibited male rat GE is mediated via a mechanism of activated CCK through its peripheral CCK1 receptor pathway. 


\section{Acknowledgements}

We thank the experimental assistance provided by Ms. JuiLing Wang.

\section{References}

1. Parkman HP, Jones MP. Tests of gastric neuromuscular function. Gastroenterology 2009;136:1526-1543.

2. Tack J, Janssen P. Gastroduodenal motility. Curr Opin Gastroenterol 2010;26:647-655.

3. Chen TS, Doong ML, Chang FY, Lee SD, Wang PS. Effects of sex steroid hormones on gastric emptying and gastrointestinal transit in rats. Am J Physiol 1995;268(1 Pt 1):G171-G176.

4. Egli M, Leeners B, Kruger TH. Prolactin secretion patterns: basic mechanisms and clinical implications for reproduction. Reproduction 2010;140:643-654.

5. Nagano M, Chastre E, Choquet A, Bara J, Gespach C, Kelly PA. Expression of prolactin and growth hormone receptor genes and their isoforms in the gastrointestinal tract. Am J Physiol 1995;268(3 Pt 1):G341-G342.

6. Chen TS, Doong ML, Wang SW, et al. Gastric emptying and gastrointestinal transit during lactation in rats. Am J Physiol 1997;272(3 Pt 1):G626-G631.

7. Williams JA. Regulation of acinar cell function in the pancreas. Curr Opin Gastroenterol 2010;26:478-483.

8. Weickert MO, Möhlig M, Spranger J, et al. Effects of euglycemic hyperinsulinemia and lipid infusion on circulating cholecystokinin. J Clin Endocrinol Metab 2008;93:2328-2333.

9. Capasso R, Izzo AA. Gastrointestinal regulation of food intake: general aspects and focus on anandamide and oleoylethanolamide. $\mathrm{J}$ Neuroendocrinol 2008;20(20 suppl 1):39-46.

10. Calogero AE, Nicolosi AM, Moncada ML, et al. Effects of cholecystokinin octapeptide on the hypothalamic-pituitary-adrenal axis function and on vasopressin, prolactin and growth hormone release in humans. Neuroendocrinology 1993;58:71-76.

11. Wu CL, Hung CR, Chang FY, Pau KY, Wang PS. Pharmacological effects of oxytocin on gastric emptying and intestinal transit of a non-nutrient liquid meal in females rats. Naunyn Schmiedeberg's Arch Pharmacol 2003;367:406-413.

12. Lv Y, Feng M, Che T, et al. CCK mediated the inhibitory effect of oxytocin on the contraction of longitudinal muscle strips of duodenum in male rats. Pflugers Arch 2010;460:1063-1071.

13. Chang FY, Lee SD, Yeh GH, Wang PS. Rat gastrointestinal motor responses mediated via activation of neurokinin receptors. J Gastroenterol Hepatol 1999;14:39-45.

14. Everett JW. Luteotropic function of autografts of the rat hypophysis. Endocrinology 1954;54:685-690.

15. Huang WJ, Yeh JY, Tsai SC, et al. Regulation of testosterone secretion by prolactin in male rats. J Cell Biochem 1999;74:111-118.

16. Doong ML, Lu CC, Kau MM, et al. Inhibition of gastric emptying and intestinal transit by amphetamine through a mechanism involving an increased secretion of CCK in male rats. Br J Pharmacol 1998; 124:1123-1130

17. Adams SA, Ajam IK, Matthews BF, Sullivan PB. Proceedings: Increased gastric emptying and intestinal motility in lactating mice. J Physiol 1976;257:57P-58P

18. Muller E, Dowling RH. Prolactin and the small intestine. Effect of hyperprolactinaemia on mucosal structure in the rat. Gut 1981;22: 558-565.

19. Gopalakrishnan V, Ramaswamy S, Pillai NP, Ghosh MN. Effect of prolactin and bromocriptine on intestinal transit in mice. Eur J Pharmacol 1981;74:369-372.

20. Vijayan E, McCann SM. The effects of the cholecystokinin antagonist, proglumide, on prolactin secretion in the rat. Life Sci 1987;40: 629-634.

21. Ebenezer IS, Thornton SN, Parrott RF. Anterior and posterior pituitary hormone release induced in sheep by cholecystokinin. Am J Physiol 1989;256(6 Pt 2):R1355-R1357.

22. Parrott RF, Ebenezer IS, Baldwin BA, Buttle HL. Intravenous administration of cholecystokinin (CCK) stimulates prolactin and growth hormone release in the pig. Methods Find Exp Clin Pharmacol 1995;17:529-533.

23. Tanimoto $\mathrm{K}$, Tamminga $\mathrm{CA}$, Chase $\mathrm{TN}$, Nilaver G. Intracerebroventricular injection of cholecystokinin octapeptide elevates plasma prolactin levels through stimulation of vasoactive intestinal polypeptide. Endocrinology 1987;121:127-132.

24. Karashima T, Okajima T, Kato K, Ibayashi H. Suppressive effects of cholecystokinin and bombesin on growth hormone and prolactin secretion in urethane-anesthetized rats. Endocrinol Jpn 1984;31:539547.

25. Miyake A, Yoshimoto Y, Kawamura Y, et al. Lunch induces an increase in the plasma prolactin concentration, but not vasoactive intestinal peptides or cholecystokinin. Endocrinol Jpn 1988;35:647650 .

26. Stengel A, Taché Y. Interaction between gastric and upper small intestinal hormones in the regulation of hunger and satiety: ghrelin and cholecystokinin take the central stage. Curr Protein Pept Sci 2011; 12:293-304.

27. Chandra R, Liddle RA. Cholecystokinin. Curr Opin Endocrinol Diabetes Obes 2007;14:63-67. 